\title{
Guava Leaf Extract Attenuates Insulin Resistance via the PI3K/Akt Signaling Pathway in a Type 2 Diabetic Mouse Model
}

This article was published in the following Dove Press journal: Diabetes, Metabolic Syndrome and Obesity: Targets and Therapy

\author{
Qiong Yang ${ }^{1, *}$ \\ Yu-Mei Wen ${ }^{1} * *$ \\ Jing Shen' \\ Mei-Mei Chen' \\ Jiang-Hua Wen ${ }^{2}$ \\ Zheng-Ming $\mathrm{Li}\left(\mathbb{D i D}^{3}\right.$ \\ Yu-Zhen Liang ${ }^{3, *}$ \\ Ning Xia ${ }^{1}, *$ \\ 'Department of Endocrinology and \\ Metabolism, The First Affiliated Hospital \\ of Guangxi Medical University, Nanning \\ 53002I, People's Republic of China; \\ ${ }^{2}$ Department of Health Examination, \\ Jiangmen Central Hospital, Jiangmen, \\ Guangdong 529070, People's Republic of \\ China; ${ }^{3}$ Department of Endocrinology \\ and Metabolism, The Second Affiliated \\ Hospital of Guangxi Medical University, \\ Nanning 53002I, People's Republic of \\ China
}

*These authors contributed equally to this work
Correspondence: Ning Xia

Department of Endocrinology and

Metabolism, The First Affiliated Hospital of Guangxi Medical University, Nanning

53002I, People's Republic of China

Tel +86 77 I2802380

Email xianinggxmu@I63.com
Background and Objective: Insulin resistance is well known to exhibit essential effects on the progression of diabetes mellitus (DM). Guava leaf was also reported to exhibit antidiabetic effects including decreasing blood glucose. Therefore, this present study aims to explore the role guava leaf extract (GLE) plays in insulin resistance and its mechanism of action via the PI3K/Akt signaling pathway.

Methods: KK-Ay mice is a spontaneous genetic type 2 diabetes mouse model induced by feeding a high fat and high sugar diet. Mice were randomly assigned into three groups: diabetic mice (DM), DM + MET (diabetic mice treated with metformin) and DM + GLE (diabetic mice treated with GLE) groups. After 8 weeks of treatment, body weight and levels of fasting plasma glucose (FPG), fasting insulin and lipids in plasma were measured. Mice were sacrificed and mRNA and protein expression of insulin receptor substrate1 (IRS1), phosphatidylinositol 3-kinase (PI3K) and serine/threonine kinase protein B (Akt) in livers were measured.

Results: GLE markedly reduced body weight, FPG, fasting insulin and insulin resistance index but increased the insulin sensitivity index of diabetic KK-Ay mice. Moreover, GLE upregulated the expression of IRS-1, PI3K and Akt mRNAs in livers of diabetic KK-Ay mice. In addition, GLE also elevated IRS-1, PI3K, Akt, p-PI3K and p-Akt protein expression in their livers. The results of the DM + MET group were similar to those of the DM + GLE group.

Conclusion: GLE plays anti-diabetic roles by ameliorating insulin resistance in KK-Ay diabetic mice and this is related to the activation of PI3K/Akt signaling pathway.

Keywords: diabetes, guava leaf extract, insulin resistance, PI3K/Akt signaling pathway

\section{Introduction}

Recently, diabetes mellitus (DM) has become a serious threat to human health all over the world. According to the International Diabetes Federation, there are 415 million adults who suffer from diabetes worldwide, and the number is rapidly increasing and will reach 642 million by 2040. ${ }^{1}$ Notably, type 2 diabetes (T2DM) accounts for about $90 \%$ of diabetic cases and is characterized by insulin resistance. ${ }^{2}$

According to the literature, the PI3K/Akt pathway is considered to participate in proliferation, differentiation, apoptosis, glucose transport and other essential biochemical processes within cells, and it was also been closely associated with insulin resistance-related T2DM. ${ }^{3,4}$ It was reported that the tyrosine phosphorylation of insulin receptor substrate (IRS) responding to insulin stimulation could activate 
phosphatidylinositol 3-kinase (PI3K), resulting in the activation of serine/threonine kinase protein $\mathrm{B}$ (Akt) and ultimately improve glucose homeostasis in the body by glucose uptake from the periphery. ${ }^{5}$ Moreover, it was also found that some drugs that upregulate PI3K/Akt signaling could effectively improve insulin sensitivity, thereby contributing to T2DM therapies. ${ }^{6,7}$

To date, only a few hypoglycemic agents, such as sulfonylureas, glinides, metformin (MET), $\alpha$-glucosidase inhibitors and SGLT2, are available for the therapy of T2DM. However, they can often exert undesirable sideeffects including weight gain, hypoglycemia, flatulence, heart failure and urinary tract infections. ${ }^{8-10}$ Given these reasons, more and more researchers have tried to find and screen more effective and safe functional components with anti-diabetic activity from some natural products.

Guava leaf, a traditional anti-inflammatory, anti-microbial, anti-oxidant and anti-diabetic herbal drug, is widely applied as a folk medicine for tropical and subtropical countries. Soman et $\mathrm{al}^{11}$ have shown that the extracts from guava leaves (GLE) could significantly decrease the levels of blood glucose, glycated hemoglobin and fructosamine in treated groups. Additionally, Shen et $\mathrm{al}^{12}$ also reported that GLE could improve hexokinase and phosphofructokinase activities in diabetic rats. In addition, the ability of cellular glucose uptake and glycogen synthesis were also significantly upregulated in the diabetic rats receiving GLE. However, the role that GLE plays in insulin resistance and its underlying mechanism are still unclear. Therefore, in our study, the role that GLE plays in diabetic KK-Ay mice and the involvement of the PI3K/Akt pathway were explored in order to clarify the biochemical mechanisms of GLE on glucose metabolism in diabetes.

\section{Materials and Methods GLE Extract}

GLE was produced and provided by Sunway Bio Co. Ltd., Guilin, Guangxi, China. In brief, the guava leaves were washed with distilled water, air-dried, crushed with a blender and then extracted with distilled water for $48 \mathrm{~h}$ at room temperature. Next, the extracts were filtered and the filtrate was subsequently lyophilized. Finally, the powder was stored at room temperature for subsequent experiments. Five kilograms of dry guava leaves were used to obtain $1 \mathrm{~kg}$ of GLE.

\section{Animals}

Eighteen KK-Ay male mice weighing $35 \pm 5 \mathrm{~g}$ (7 weeks) were provided by Beijing HFK Bioscience Co. Ltd. Mice were kept within climate-controlled housing $\left(23 \pm 2^{\circ} \mathrm{C}\right)$ with $50 \%$ relative humidity and a $12 \mathrm{~h} \mathrm{light/dark} \mathrm{cycle.} \mathrm{All}$ experimental protocols were approved by the Institutional Animal Care and Use Committees of Guangxi Medical University, and experiments were performed based on the guidance on the use of laboratory animals from the National Institute of Health.

The KK-Ay mice were fed with commercial KK mice diet (Product ID: 1042, HFK Bioscience Co. Ltd.) containing 17.5 protein, $48.5 \%$ carbohydrate and $17.9 \%$ fat. Water was available ad libitum. A fasting glucose concentration $\geq 13$. $9 \mathrm{mmol} / \mathrm{L}$ was defined as a stable diabetic state. Diabetic mice were randomly assigned into three groups (6 mice per group) for an 8-week feeding experiment: diabetic mice (DM), DM + MET (diabetic mice treated with metformin) and DM + GLE (diabetic mice treated with GLE) groups. Each mouse in the DM + MET group was intra-gastrically administrated with MET $(227.5 \mathrm{mg} /$ kg/day; H20023371; Sino-American Shanghai Squibb Pharmaceutical Co. Ltd). Each mouse in the DM + GLE group was intra-gastrically administrated with GLE $(1638 \mathrm{mg} / \mathrm{kg} / \mathrm{day})$. For the DM group, equal amounts of saline were intra-gastrically given to each mouse based on the same procedure. There were no adverse effects in the mice receiving metformin and GLE treatments.

\section{Measurement of Physiological and Biochemical Indexes}

Body weight and blood glucose levels were monitored every week. After 8 weeks of treatment, mice were killed. Blood samples were harvested from the orbital sinus and immediately centrifuged for $10 \mathrm{~min}$ at $4^{\circ} \mathrm{C}$ to obtain the serum. The hepatic tissues were also collected and then stored at $-80^{\circ} \mathrm{C}$ for further use. FPG levels were determined with a glucometer (Roche Diagnostic $\mathrm{GmbH}$, Germany). The levels of total cholesterol (TC), triglycerides (TG), low-density lipoprotein cholesterol (LDL-C) and high-density lipoprotein cholesterol (HDL-C) in serum were determined with a blood chemistry analyzer (Hitachi 7600).

Fasting insulin levels were measured based on the manufacturer's instructions using a mouse insulin enzyme-linked immunosorbent assay kit (Cusabio Technology Co. Ltd., Wuhan, China). Homeostasis model assessment of insulin resistance (HOMA-IR), and insulin sensitivity index (ISI) were calculated based on the following formulae, respectively: 
HOMA-IR $=$ fasting insulin $(\mathrm{mIU} / \mathrm{L}) \times \mathrm{FPG}(\mathrm{mmol} / \mathrm{L})] / 22.5$; $\mathrm{ISI}=1 /[$ fasting insulin $(\mathrm{mIU} / \mathrm{L}) \times \mathrm{FPG}(\mathrm{mmol} / \mathrm{L})]$.

\section{RT-PCR}

The expression of IRS-1, PI3K and Akt mRNAs were detected using real-time PCR (RT-PCR). Total RNA from hepatic tissues were isolated using the TRIzol reagent (Takara Bio, Dalian, China). The total RNA was reversely transcribed to cDNA using a PrimeScript RT Reagent Kit with gDNA Eraser (Takara Bio, Dalian, China). Subsequently, gene expression was quantitatively measured using RT-PCR with SYBR Green reagent (Toyobo, Japan) in an ABI 7500 System (Applied Biosystems, USA). The relative mRNA expression levels of IRS-1, PI3K and Akt were calculated using the $2^{-\triangle \Delta C T}$ method, and $\beta$-actin was used as a housekeeping gene. The detailed information for each primer sequence are provided in Table 1.

\section{Western Blots}

Total protein was extracted from hepatic tissues, separated with SDS-PAGE (10\% to $15 \%)$, and then transferred onto PVDF (Millipore, USA). Subsequently, the membranes were incubated with non-fat milk for $1.5 \mathrm{~h}$ at room temperature and then incubated overnight at $4{ }^{\circ} \mathrm{C}$ with primary anti-IRS-1, antiPI3K, anti-Akt, anti-p-PI3K, anti-p-Akt or anti- $\beta$-actin antibodies (1: 500; Cell Signaling Technology). After 5 washes with TBST, the membranes were incubated with secondary antibodies for $2 \mathrm{~h}$ at room temperature. Finally, an enhanced chemiluminescence detection system (Pierce, Rockford, IL, USA) and the Image Lab software Bio-Rad were used to detect and quantify the bands for protein expression.

\section{Statistical Analysis}

SPSS 16.0 software was used for statistical analysis. The data are shown as mean \pm SD. One-way ANOVA or

Table I Primers for RT-PCR

\begin{tabular}{|l|l|l|l|}
\hline Gene & Primer & Primer Sequences (5' to $\mathbf{3}^{\prime}$ ) & Size (bp) \\
\hline AKT & $\begin{array}{l}\text { Forward } \\
\text { Reverse }\end{array}$ & $\begin{array}{l}\text { TGCACAAACGAGGGGAATATAT } \\
\text { CGTTCCTTGTAGCCAATAAAGG }\end{array}$ & 88 \\
\hline PI3K & $\begin{array}{l}\text { Forward } \\
\text { Reverse }\end{array}$ & $\begin{array}{l}\text { AAACAAAGCGGAGAACCTATTG } \\
\text { TAATGACGCAATGCTTGACTTC }\end{array}$ & 125 \\
\hline IRS-I & $\begin{array}{l}\text { Forward } \\
\text { Reverse }\end{array}$ & $\begin{array}{l}\text { GAGTTGAGTTGGGCAGAATAGG } \\
\text { CCTATCTGCATGGTCATGTAGT }\end{array}$ & 109 \\
\hline $\begin{array}{l}\text { Forward } \\
\text { Reverse }\end{array}$ & $\begin{array}{l}\text { CTACCTCATGAAGATCCTGACC } \\
\text { CACAGCTTCTCTTTGATGTCAC }\end{array}$ & 90 \\
\hline
\end{tabular}

repeated-measures ANOVA was used to analyze the significant differences among the three groups. The means of every two groups were detected using LSD. P values of less than 0.05 were considered as significant.

\section{Results}

\section{Effects of GLE on Body Weight of Diabetic KK-Ay Mice}

As shown in Table 2, after 8 weeks of GLE treatment, statistically significant differences were observed in body weight among the three groups. Compared with the DM +MET group and DM group, the body weight was markedly decreased in the DM+GLE group (both $\mathrm{P}<0.05$ ). However, there no significant differences in body weight between the DM + GLE and DM+MET groups $(\mathrm{P}>0.05)$.

\section{Effects of GLE on Serum Lipid Parameters}

After 8 weeks of treatment, the serum levels of TC, TG, LDL-C and HDL-C were measured and are shown in Table 3. Compared with the DM group, GLE treatment slightly reduced TG and LDL-C levels and slightly enhanced TC and HDL-C levels, but there were no significant differences in the levels of TC, TG, LDL-C and HDL-C between the 3 groups $(\mathrm{P}>0.05)$.

\section{Effects of GLE on FPG, HOMA-IR and ISI of Diabetic KK-Ay Mice}

As shown in Table 4, compared to the DM group, the FPG and HOMA-IR significantly decreased while the ISI increased

Table 2 Effects of GLE on Body Weight of KK-Ay Mice

\begin{tabular}{|l|l|l|l|l|}
\hline Weeks & DM (n=6) & $\begin{array}{l}\text { DM + MET } \\
(\mathbf{n = 6})\end{array}$ & $\begin{array}{l}\text { DM + GLE } \\
(\mathbf{n = 6})\end{array}$ & F \\
\hline 0 & $36.87 \pm 1.43$ & $36.63 \pm 2.76$ & $36.92 \pm 2.76$ & 0.034 \\
4 & $39.05 \pm 3.18$ & $38.2 \pm 4.16$ & $36.92 \pm 4.27$ & 1.006 \\
8 & $39.77 \pm 2.00$ & $37.15 \pm 1.13$ & $32.52 \pm 3.59^{\mathrm{a},}$ & 13.848 \\
& & & $\mathrm{~b}$ & \\
\hline
\end{tabular}

Notes: ${ }^{a} P<0.05$ vs DM group ${ }^{b} P<0.05$ vs DM + MET group.

Table 3 Effects of GLE on Serum Lipids of KK-Ay Mice

\begin{tabular}{|l|l|l|l|l|}
\hline Lipids & DM (n=6) & $\begin{array}{l}\text { DM + MET } \\
(\mathbf{n}=6)\end{array}$ & $\begin{array}{l}\text { DM + GLE } \\
(\mathbf{n}=6)\end{array}$ & F \\
\hline HDL-C (mmol/L) & $3.40 \pm 0.91$ & $3.30 \pm 0.81$ & $3.83 \pm 0.69$ & 0.732 \\
LDL-C (mmol/L) & $0.48 \pm 0.23$ & $0.30 \pm 0.20$ & $0.26 \pm 0.13$ & 2.177 \\
TC (mmol/L) & $5.16 \pm 0.90$ & $6.08 \pm 3.45$ & $5.48 \pm 1.02$ & 0.290 \\
TG (mmol/L) & $5.46 \pm 1.17$ & $4.30 \pm 2.06$ & $4.38 \pm 0.91$ & 1.176 \\
\hline
\end{tabular}


Table 4 Effects of GLE on FPG and Fasting Insulin Levels as Well as HOMA-IR and ISI of Diabetic KK-Ay Mice

\begin{tabular}{|l|l|l|l|l|}
\hline Variables & DM (n=6) & DM + MET (n=6) & DM + GLE (n=6) & F \\
\hline FPG (mmol/L) & $21.35 \pm 2.39$ & $12.57 \pm 2.45^{\mathrm{a}}$ & $13.80 \pm 1.75^{\mathrm{a}}$ & 27.572 \\
Fasting insulin (mU/L) & $20.87 \pm 7.28$ & $17.57 \pm 2.52$ & $13.62 \pm 1.92^{\mathrm{a}}$ & 3.753 \\
HOMA-IR & $2.94 \pm 0.36$ & $2.26 \pm 0.34^{\mathrm{a}}$ & $2.11 \pm 0.26^{\mathrm{a}}$ & 11.336 \\
ISI & $0.0025 \pm 0.0008$ & $0.0049 \pm 0.0017^{\mathrm{a}}$ & $0.0056 \pm 0.0015^{\mathrm{a}}$ & 8.505 \\
\hline
\end{tabular}

Note: ${ }^{a} P<0.05$ vs DM group.

significantly in both the DM + GLE and DM+MET groups after 8 weeks of treatment $(P<0.05)$. Fasting insulin was also significantly decreased in the DM + GLE group $(P<0.05)$. However, there no significant differences in FPG and fasting insulin levels as well as HOMA-IR and ISI observed between the DM + GLE and DM+MET groups $(P>0.05)$.

\section{Effects of GLE on mRNA and Protein Expression Levels of IRS-I, PI3K and Akt in Livers of Diabetic KK-Ay Mice}

The expression levels of IRS-1, PI3K and Akt mRNAs in livers of both the DM + MET and DM + GLE groups were markedly enhanced compared to those of the DM group $(P<0.05$; Figure 1). Moreover, the expression levels of IRS-1, PI3K, Akt, p-PI3K and p-Akt proteins were also obviously upregulated in both of the treatment groups compared with those of the DM group $(P<0.05$; Figure 2). However, there no significant differences in the gene and protein expressions of these intermediates of the PI3K/Akt signaling pathway between the DM + GLE and DM + MET groups.

\section{Discussion}

Our study showed that GLE treatment decreased FPG levels and body weight by alleviating insulin resistance, thereby ameliorating T2DM. Additionally, these effects might be mediated through the activated PI3K/Akt signaling pathway and IRS1 expression in the livers of T2DM mice.

Modern pharmacology showed that guava leaves contain phytochemicals with hypoglycemic properties, such as flavonoids, phenolic acids, triterpenes and sesquiterpenes. ${ }^{13}$ Shen et $\mathrm{al}^{12}$ reported that long-term feeding of GLE could significantly reduce the FPG levels of T2DM rats. Similarly, the study reported by Cheng et $\mathrm{al}^{14}$ also found that GLE could promote the absorption of glucose through hepatocytes, which in turn can contribute to the reduction of hyperglycemia in diabetic patients. Our study was consistent with their conclusions, demonstrating that GLE exhibited anti-hyperglycemic activity. Specifically, after 8 weeks of treatment, compared to the DM group, the FPG and fasting insulin levels and the HOMA-IR of diabetic KKAy mice significantly decreased, while the ISI significantly increased in the DM + GLE group, suggesting that GLE could alleviate insulin resistance. There were no significant differences in FPG and fasting insulin levels as well as HOMA-IR and ISI observed between the DM + GLE and $\mathrm{DM}+\mathrm{MET}$ groups indicating that GLE might exhibit similar effects of decreasing glucose and improving insulin resistance with MET but with relatively fewer side-effects.

$\mathrm{PI} 3 \mathrm{~K} / \mathrm{Akt}$ is a major downstream signaling pathway of insulin and plays key roles in many physiological and pathological processes such as cell survival, differentiation
A

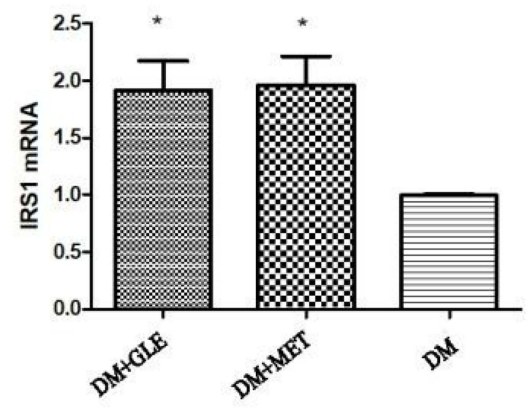

B

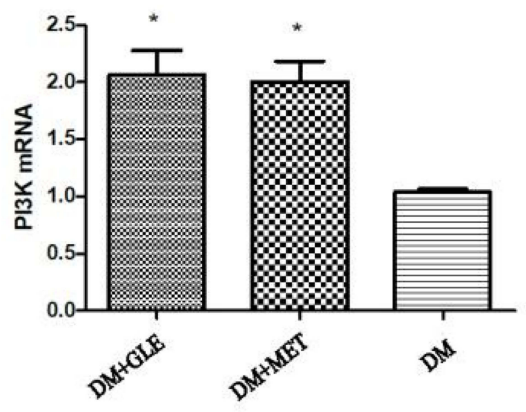

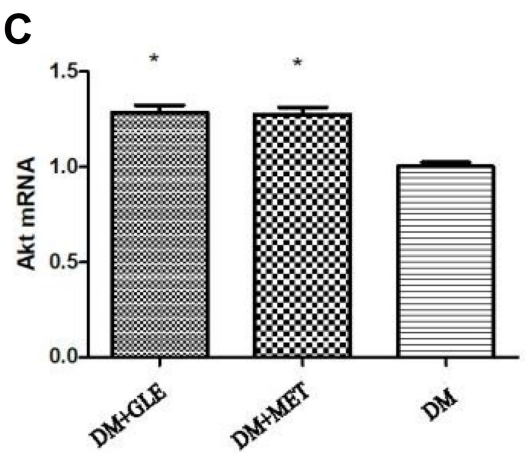

Figure I Effects of GLE on the expression levels of IRS-I, PI3K and Akt mRNAs in livers of diabetic KK-Ay mice. (A) IRS-I. (B) PI3K. (C) Akt. *P < 0.05 vs DM group. 
A

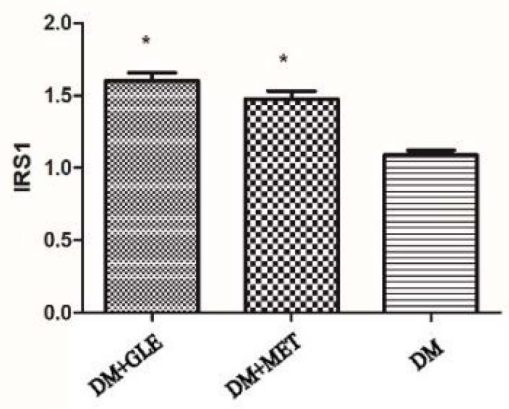

D

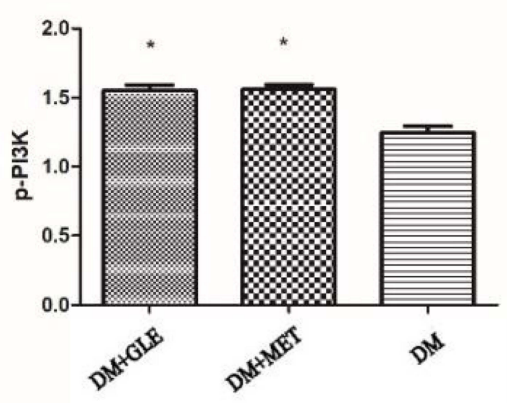

B

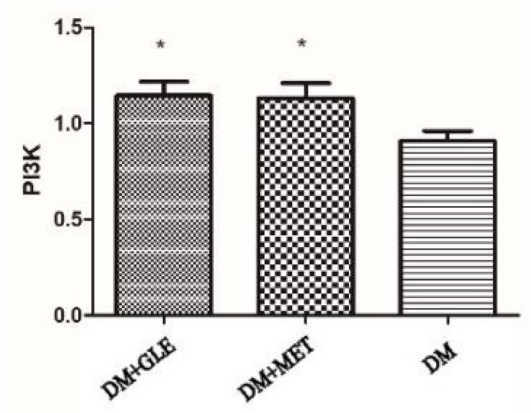

E

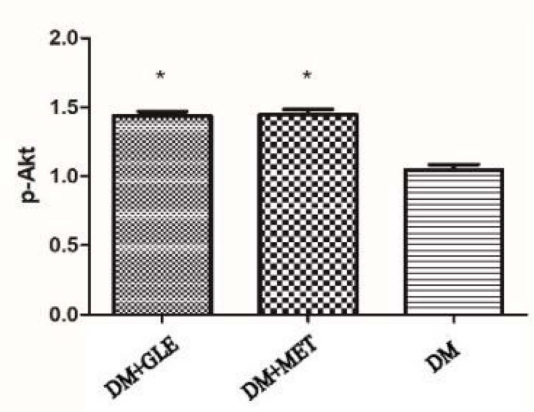

C

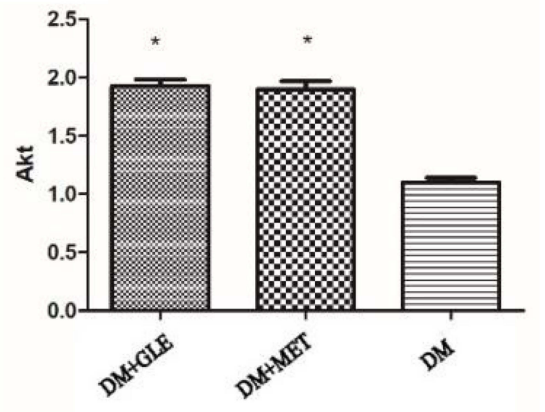

$\mathbf{F}$

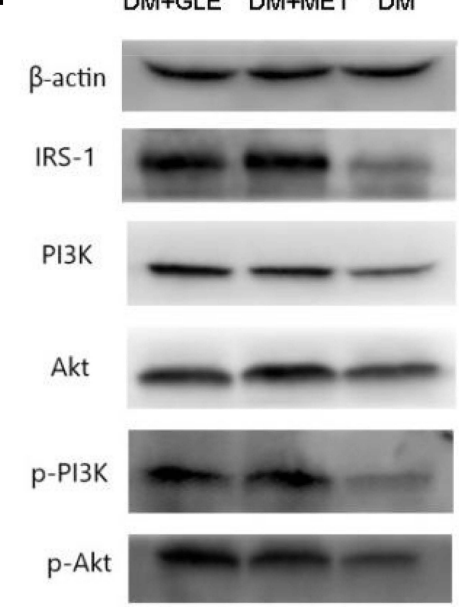

Figure 2 Effects of GLE on the expression levels of IRS-I, PI3K, Akt, p-PI3K and p-Akt proteins in livers of diabetic KK-Ay mice. (A) IRS-I. (B) PI3K. (C) Akt. (D) P-PI3K. (E) p-Akt. (F) Representative images for Western blots. $* P<0.05$ vs DM group.

and glucose metabolism. ${ }^{15,16}$ Insulin mainly binds to the $\alpha$-subunit of insulin receptors in livers, skeletal muscles and adipose tissues, thereby activating the tyrosine phosphorylation of IRS-1. Subsequently, the phosphorylated IRS-1 binds to p85, a regulatory subunit of PI3K, which in turn leads to the phosphorylation of Akt and glycogen synthase kinase 3 (GSK3), which could affect glucose metabolism by regulating glycogen synthesis, gluconeogenesis and glucose transport. ${ }^{17}$

Natural products have a long history of being used as antidiabetic drugs. Previous studies have reported that berberine extracted from Coptis chinensis Franch can increase insulininduced IRS-1 tyrosine phosphorylation and the recruitment of p85 to IRS-1, suggesting that berberine may alleviate insulin resistance through regulating some important molecules in the PI3K/Akt signaling pathway. ${ }^{18}$ However, the anti-diabetic action mechanism of GLE is still not fully clarified to date. Previous studies reported that the anti-diabetic action mechanism of GLE is mainly related to: (1) the decreases in body weight and blood lipid metabolism; ${ }^{19,20}$ (2) the inhibition of $\alpha$-glucosidase ${ }^{21}$ (3) the promotion of liver glycogen synthesis; ${ }^{22,23}$ and (4) the inhibition of dipeptidyl peptidase $\mathrm{IV}^{24}$ To further explore the potential mechanism of action of GLE in ameliorating T2DM, our study determined the involvement of the PI3K/Akt signaling pathway. We found that the mRNA and protein expression levels of IRS-1, PI3K and Akt, as well as the p-PI3K and p-Akt levels in livers of diabetic KK-Ay mice in the DM + GLE group were much higher than those in the DM group. These results suggested that GLE might play an anti-hyperglycemic role by upregulating the PI3K/Akt signaling pathway.

In conclusion, our results showed that GLE exhibits an anti-hyperglycemic effect on diabetic KK-Ay mice by activating the PI3K/Akt signaling pathway and subsequently ameliorating insulin resistance. These results provide a theoretical basis for the application of GLE for the prevention and therapy of T2DM as well as the insulin resistance-related metabolic syndrome.

Although this study has addressed liver insulin resistance by studying the alterations in PI3K/Akt signaling, future 
studies should focus on other molecular mechanisms central to hepatic gluconeogenesis. Similarly, future studies are warranted to address pathway-specific insulin resistance amelioration by GLE in skeletal muscle which is one of the most important target organs of insulin action. The safety parameters such as liver and renal enzyme measurements should also be assessed in future work so as to emphasize the non-toxic nature and safety level of the natural product.

\section{Acknowledgments}

The project was supported by the Natural Science Foundation of Guangxi Province (2017GXNSFAA198198). The authors would like to thank Dr. Dev Sooranna, Imperial College London, for editing the manuscript.

\section{Disclosure}

All authors report no conflicts of interest in this work.

\section{References}

1. Vinayagam R, Xiao J, Xu B. An insight into anti-diabetic properties of dietary phytochemicals. Phytochem Rev. 2017;16(3):535-553. doi:10.1007/s11101-017-9496-2

2. Hogger P, Xiao JB. Dietary polyphenols and type 2 diabetes: current insights and future perspectives. Curr Med Chem. 2015;22(1):23-38. doi:10.2174/0929867321666140706130807

3. Manning BD, Toker A. AKT/PKB Signaling: navigating the Network. Cell. 2017;169(3):381-405. doi:10.1016/j.cell.2017.04.001

4. Saini KS, Loi S, De Azambuja E, et al. Targeting the PI3K/AKT/mTOR and Raf/MEK/ERK pathways in the treatment of breast cancer. Cancer Treat Rev. 2013;39(8):935-946. doi:10.1016/j.ctrv.2013.03.009

5. Carvalho E, Rondinone C, Smith U. Insulin resistance in fat cells from obese Zucker rats - Evidence for an impaired activation and translocation of protein kinase B and glucose transporter 4. Mol Cell Biochem. 2000;206:7-16. doi:10.1023/A:1007009723616

6. Chan SMH, Ye JM. Strategies for the discovery and development of anti-diabetic drugs from the natural products of traditional medicines. J Pharm Pharm Sci. 2013;16(2):207-216. doi:10.18433/J3T60G

7. $\mathrm{Hu} \mathrm{X}$, Wang $\mathrm{M}$, Bei W, et al. The Chinese herbal medicine FTZ attenuates insulin resistance via IRS1 and PI3K in vitro and in rats with metabolic syndrome. $J$ Transl Med. 2014;12:47. doi:10.1186/ 1479-5876-12-47

8. Stein SA, Lamos EM, Davis SN. A review of the efficacy and safety of oral antidiabetic drugs. Expert Opin Drug Saf. 2013;12(2):153-175. doi:10.1517/14740338.2013.752813

9. Bailey CJ, Day C. The future of new drugs for diabetes management. Diabetes Res Clin Pract. 2019;155:107785. doi:10.1016/j.diabres. 2019.107785
10. Vieira R, Souto SB, Sanchez-Lopez E, et al. Sugar-lowering drugs for type 2 diabetes mellitus and metabolic syndrome-review of classical and new compounds: part-I. Pharmaceuticals (Basel). 2019;12:152. doi: $10.3390 / \mathrm{ph} 12040152$

11. Soman S, Rauf AA, Indira M, et al. Antioxidant and antiglycative potential of ethyl acetate fraction of psidium guajavaleaf extract in streptozotocin-induced diabetic Rats. Plant Foods Hum Nutr. 2010;65(4):386-391. doi:10.1007/s11130-010-0198-9

12. Shen SC, Cheng FC, Wu NJ. Effect of guava (Psidium guajava Linn.) leaf soluble solids on glucose metabolism in type 2 diabetic rats. Phytother Res. 2008;22(11):1458-1464. doi:10.1002/ptr.2476

13. Gutiérrez RM, Mitchell S, Solis RV. Psidium guajava: a review of its traditional uses, phytochemistry and pharmacology. J Ethnopharmacol. 2008;117(1):0-27.

14. Cheng FC, Shen SC, Wu JS. Effect of guava (Psidium guajava L.) leaf extract on glucose uptake in rat hepatocytes. J Food Sci. 2009;74 (5):H132-H138. doi:10.1111/j.1750-3841.2009.01149.x

15. Alessandro $B$, Federica $C$, Geltrude $M$, et al. Insulin signaling in insulin resistance states and cancer: a modeling analysis. PLoS One. 2016;11(5):e0154415. doi:10.1371/journal.pone.0154415

16. Du Y, Wei T. Inputs and outputs of insulin receptor. Protein Cell. 2014;5(3):203-213. doi:10.1007/s13238-014-0030-7

17. Dokken BB, Sloniger JA, Henriksen EJ. Acute selective glycogen synthase kinase-3 inhibition enhances insulin signaling in prediabetic insulin-resistant rat skeletal muscle. Am J Physiol Endocrinol Metab. 2005;288(6):1188-1194. doi:10.1152/ajpendo.00547.2004

18. Liu LZ, Cheung SCK, Lan LL, et al. Berberine modulates insulin signaling transduction in insulin-resistant cells. Mol Cell Endocrinol. 2010;317(1-2):148-153. doi:10.1016/j.mce.2009.12.027

19. Attawish A, Chavalittumrong P, Rugsamon P, et al. Toxicity study of Psidium guajava Linn. leaves. Bull Dep Med Sci. 2013.

20. Mathur R, Shagun D, Velpandian T, et al. Psidium guajavaLinn. leaf extract affects hepatic glucose transporter-2 to attenuate early onset of insulin resistance consequent to high fructose intake: an experimental study. Pharmacognosy Res. 2015;7(2):166-175. doi:10.4103/09748490.151459

21. Wang L, Liu Y, Luo Y, et al. Quickly screening for potential $\alpha$ glucosidase inhibitors from guava leaves tea by bioaffinity ultrafiltration coupled with HPLC-ESI-TOF/MS method. J Agr Food Chem. 2018;66(6):acs.jafc.7b05280.

22. Vinayagam R, Jayachandran M, Chung S, et al. Guava leaf inhibits hepatic gluconeogenesis and increases glycogen synthesis via AMPK/ ACC signaling pathways in streptozotocin-induced diabetic rats. Biomed Pharmacother. 2018;103(2018). doi:10.1016/j.biopha.2018.04.127

23. Liu CW, Wang YC, Hsieh CC, et al. Guava (Psidium guajava Linn.) leaf extract promotes glucose uptake and glycogen accumulation by modulating the insulin signaling pathway in high-glucose-induced insulin-resistant mouse FL83B cells. Proc Biochem. 2015;50 (7):1128-1135. doi:10.1016/j.procbio.2015.03.022

24. Eidenberger T, Selg M, Krennhuber K. Inhibition of dipeptidyl peptidase activity by flavonol glycosides of guava (Psidium guajava L.): a key to the beneficial effects of guava in type II diabetes mellitus. Fitoterapia. 2013;89(Complete):74-79. doi:10.1016/j.fitote.2013.05.015

\section{Publish your work in this journal}

Diabetes, Metabolic Syndrome and Obesity: Targets and Therapy is an international, peer-reviewed open-access journal committed to the rapid publication of the latest laboratory and clinical findings in the fields of diabetes, metabolic syndrome and obesity research. Original research, review, case reports, hypothesis formation, expert opinion and commentaries are all considered for publication. The manuscript management system is completely online and includes a very quick and fair peer-review system, which is all easy to use. Visit http://www.dovepress.com/testimonials.php to read real quotes from published authors. 